Journal of Theoretical and Applied Mechanics, Sofia, 2016, vol. 46, No. 1, pp. 83-98

\title{
ELECTRIC AND HEAT CONDUCTIONS ACROSS A CRACK IN A THERMOELECTRIC MATERIAL*
}

\author{
HaO-Peng Song, Kun Song \\ State Key Laboratory of Mechanics and Control of Mechanical Structures, \\ Nanjing University of Aeronautics \& Astronautics, Nanjing 210016, China, \\ e-mail: shp305@163.com
}

[Received 24 August 2015. Accepted 29 February 2016]

\begin{abstract}
The crack problem in a thermoelectric material is studied in this paper. Two kinds of crack surface conditions are discussed. The closed form solutions are derived, based on the complex variable method. The field intensity factors as well as the conversion efficiency are discussed in detail. The results show that the electric current density and thermal flux density exhibit traditional square-root singularity at the crack tip in case 1 , while the electric current density has no singularity at the crack tip in case 2. It is proved due to the electric current flow and thermal flux separation, that the thermoelectric conversion efficiency can be higher than the maximum conversion efficiency of one-dimensional thermoelectric under the same temperature condition. In the numerical example, the conversion efficiency is increased by $29.6 \%$ as compared to the maximum conversion efficiency of one-dimensional thermoelectric.

KEY WORDS: Thermoelectric material, crack, field intensity factor, conversion efficiency.
\end{abstract}

\section{Introduction}

Thermoelectric materials are widely pursued for technological applications due to intrinsic coupling behaviour, including waste heat recovery, solid state thermal management, solar energy harvesting and carbon reduction (Disalvo [1]; Yang and Caillat [2]; Narducci [3]; Tritt and Subramanian [4]; Kraemer et al [5]; Bell [6]). Much effort has been devoted to developing hybrid thermoelectric materials with high conversion efficiency (Heremans et al [7]; Snyder

\footnotetext{
* Corresponding author e-mail: shp305@163.com

The work was supported by National Natural Science Foundation of China (Nos. 11232007, 11202099, 11172255), and funded by PAPD, State Key Laboratory of Mechanics and Control of Mechanical Structures (MCMS-0215G01), NSF (CMMI-1235535).
} 
and Toberer [8]; Gothard et al [9]; Vashaee and Shakouri [10]). Though continuum analysis of thermoelectrics has only recently attracted some attentions (Webman et al [11]; Mahan [12]), despite significant progress has been made in thermoelectric material development.

Most of the investigations on thermoelectric crack problems were experimental in nature. For example, Isoda et al [13] tested the thermal shock resistance and thermo electric properties of boron doped iron disilicides. The grain-boundary crack, appeared in the initial period of thermal duration test of $\mathrm{CoSb}_{3}$ thermoelectric material, was observed by Zhao et al [14]. Schmidt et al [15] studied the room-temperature mechanical properties and slow crack growth behaviour of $\mathrm{Mg}_{2} \mathrm{Si}$, and Eilertsen et al [16] explored the fracture toughness of $\mathrm{Co}_{4} \mathrm{Sb}_{12}$ and $\mathrm{In}_{0.1} \mathrm{Co}_{4} \mathrm{Sb}_{12}$ thermoelectric skutterudites by three methods. These studies highlighted the importance of thermoelectric reliability, yet, the analysis of the effectiveness of cracks on the conversion efficiency, is rare.

The main effort on improving the conversion efficiency is improving the thermoelectric figure of merit. For example, it was reported that the $\mathrm{Ag}_{1-x} \mathrm{~Pb}_{18} \mathrm{SbTe}_{20}$ showed a very high $Z T$ value of 2.2 at $800 \mathrm{~K}$ [17], and with grain size less than $100 \mathrm{~nm}$, the $Z T$ value of $\mathrm{Bi}_{x} \mathrm{Sb}_{2-x} \mathrm{Te}_{3}$ nano-composite is increased from 1.2 to 1.4 [18], at about $370 \mathrm{~K}$. A Bi $\mathrm{Be}_{3} / \mathrm{Sb}_{2} \mathrm{Te}_{3}$ bulk nanocomposites with laminated nanostructures was produced, by using a simple route involving hydrothermal synthesis and hot pressing, which has a maximum $Z T$ value of 1.4 at $450 \mathrm{~K} \mathrm{[19].} \mathrm{Whether} \mathrm{there} \mathrm{is} \mathrm{another} \mathrm{way} \mathrm{to} \mathrm{improve}$ the conversion efficiency of the thermoelectric material? This fact motivated our current work.

In the present work, the crack problem in a thermoelectric material is studied. The general solution for two-dimensional problem is derived, then the analytical solutions for a crack embedded in an infinite thermoelectric matrix are presented and finally, discussions are made about the effects of crack on the conversion efficiency of the thermoelectric material.

\section{Governing equations}

Since the material properties do not change in the unit cell, the coupled transports of heat and electrons in a thermoelectric material, with the respective transport equations, are given by (Harman and Honig [20]):

$$
\begin{gathered}
-\mathbf{J}_{N}=\left(\frac{T \sigma}{e^{2}}\right) \frac{1}{T} \nabla \mu-\left(\frac{T^{2} \sigma \varepsilon}{e}\right) \nabla \frac{1}{T}, \\
\mathbf{J}_{Q}=-\left(\frac{T^{2} \sigma \varepsilon}{e}\right) \frac{1}{T} \nabla \mu+\left(T^{3} \sigma \varepsilon^{2}+T^{2} \kappa\right) \nabla \frac{1}{T},
\end{gathered}
$$


wherein the electron flux $\mathbf{J}_{N}$ is coupled with the heat flux $\mathbf{J}_{Q}$ through the seebeck coefficient, and $T, \sigma, \kappa, e$ and $\mu$ are the temperature, electric conductivity, thermal conductivity, charge and electrochemical potential of the electron, respectively. Note, that:

$$
\mu=e \varphi,
$$

where $\varphi$ is the electric potential. Since the electric current density can be derived from electron flux:

$$
\mathbf{J}=e \mathbf{J}_{N} .
$$

The transport equations governing electric current density and heat flux can be derived as follows:

$$
\begin{gathered}
-\mathbf{J}=\sigma \nabla \varphi+\sigma \varepsilon \nabla T, \\
\mathbf{J}_{Q}=T \varepsilon \mathbf{J}-\kappa \nabla T .
\end{gathered}
$$

Since energy is transported by both electrons and heat, the energy flux $\mathbf{J}_{E}$ can be expressed as:

$$
\mathbf{J}_{E}=\mathbf{J}_{Q}+\varphi \mathbf{J} .
$$

In the following analysis, we limit ourselves to systems wherein both charges and energy are conserved, such that both current density and energy flux are divergence-free (Yang, [21]):

$$
\begin{gathered}
\nabla \cdot \mathbf{J}=0, \\
\nabla \cdot\left(\mathbf{J}_{Q}+\varphi \mathbf{J}\right)=0 .
\end{gathered}
$$

Notice, that the heat flux is not divergence free due to Joule heating.

\section{General solutions of two-dimensional problems}

For the case of two-dimensional problems, the fields of temperature, electric potential, heat flow and electric current can be expressed by two analytic complex functions $f(z)$ and $f_{2}(z)$. To this end, we substitute Eq. (5) into Eq. (8) to obtain:

$$
\nabla^{2}(\varphi+\varepsilon T)=0
$$


According to Muskhelishvili [22], let $\varphi+\varepsilon T$ be the real part of the analytic complex function $f(z)$, we have:

$$
\operatorname{Re}[f(z)]=\varphi+\varepsilon T,
$$

where $z=x+i y$.

From Eq. (5), the current density can be represented as:

$$
\begin{aligned}
& J_{x}=-\frac{1}{2} \sigma \frac{\partial(f(z)+\overline{f(z)})}{\partial x \overline{f(z)}}=-\frac{\sigma}{2}\left(f^{\prime}(z)+\overline{f^{\prime}(z)}\right), \\
& J_{y}=-\frac{1}{2} \sigma \frac{\partial i}{\partial y}\left(f^{\prime}(z)-\overline{f^{\prime}(z)}\right),
\end{aligned}
$$

or

$$
J_{x}-i J_{y}=-\sigma f^{\prime}(z), \quad J_{r}-i J_{\theta}=-\sigma e^{i \theta} f^{\prime}(z) .
$$

Substituting Eqs (1-2) into Eq. (9), we have:

$$
\nabla \cdot(T \varepsilon \mathbf{J}-\kappa \nabla T)+\mathbf{J} \nabla \varphi+\varphi \nabla \cdot \mathbf{J}=0 .
$$

Noting, that $\nabla \cdot \mathbf{J}=0$, Eq. (14) can be rewritten as:

$$
\mathbf{J}^{2}=-\sigma \kappa \nabla^{2} T=\frac{\sigma \kappa \nabla^{2} \varphi}{\varepsilon} .
$$

Combining it with Eqs (5) and (11), we obtain:

$$
\frac{\mathbf{J}^{2}}{\sigma^{2}}=[\nabla \operatorname{Re}[f(z)]]^{2}=-\frac{\kappa \nabla^{2} T}{\sigma}=\frac{\kappa \nabla^{2} \varphi}{\sigma \varepsilon} .
$$

Namely:

$$
\begin{aligned}
& \nabla^{2} T=-\frac{\sigma}{\kappa}[\nabla \operatorname{Re}[f(z)]]^{2}=-\frac{\sigma}{\kappa} \frac{\partial f(z)}{\partial z} \frac{\partial \overline{f(z)}}{\partial \bar{z}} \\
& \nabla^{2} \varphi=\frac{\sigma \varepsilon}{\kappa} \frac{\partial f(z)}{\partial z} \frac{\partial \overline{f(z)}}{\partial \bar{z}} .
\end{aligned}
$$

Integrating Eq. (17), we have:

$$
\begin{gathered}
T=-\frac{\sigma}{4 \kappa} f(z) \overline{f(z)}+f_{2}(z)+\overline{f_{2}(z)}+C_{2}, \\
\varphi=\frac{\sigma \varepsilon}{4 \kappa} f(z) \overline{f(z)}+\frac{1}{2}[f(z)+\overline{f(z)}]-\varepsilon\left[f_{2}(z)+\overline{f_{2}(z)}\right]-\varepsilon C_{2},
\end{gathered}
$$


where $C_{2}$ is real and denotes an uniform temperature field.

The thermal flux can then be derived from Eq. (6), as:

$$
\begin{aligned}
J_{Q x}-i J_{Q y}=\frac{\sigma^{2} \varepsilon}{4 \kappa} f(z) \overline{f(z)} & f^{\prime}(z)-\sigma \varepsilon C_{2} f^{\prime}(z) \\
& +\frac{\sigma f^{\prime}(z) \overline{f(z)}}{2}-2 \sigma \varepsilon f^{\prime}(z) \operatorname{Re}\left[f_{2}(z)\right]-2 \kappa f_{2}^{\prime}(z),
\end{aligned}
$$

where $f_{2}(z)$ is any analytic complex function.

As such, if the two analytic complex functions $f(z)$ and $f_{2}(z)$ can be determined, the fields of temperature, electric potential, heat flow and electric current can be derived.

\section{Solutions of crack problem}

Now, consider an open line segment on $x$ axis $S=(-a, a)$ embedded in an infinite thermoelectric material. The material is subjected to in-plane electric current $J_{x}^{\infty}, J_{y}^{\infty}$ and heat flow $J_{Q x}^{\infty}, J_{Q y}^{\infty}$ at infinity, as shown in Fig. 1. (a). It should be pointed out that the electric current $J_{x}^{\infty}, J_{y}^{\infty}$ also produce heat flow $J_{Q x}^{\infty \prime}, J_{Q y}^{\infty \prime}$ at infinity. Here, the heat flows $J_{Q x}^{\infty}$ and $J_{Q y}^{\infty}$ stand for the thermal loads, which are independent of the electric current. Therefore, the total heat flows at infinity are $J_{Q x}^{\infty \prime}+J_{Q x}^{\infty}$ and $J_{Q y}^{\infty \prime}+J_{Q y}^{\infty}$. All the material properties and field variables are assumed to be only dependent on coordinates $x$ and $y$.

\subsection{Crack surface conditions}

Here we will discuss two crack surface conditions:

Case 1, thermally and electrically impermeable crack. Similar impermeable crack boundary conditions has been used in elastic material (Irwin [23]) and piezoelectric materials (Deeg [24] and Park [25]). In thermoelectric materials, impermeable boundary condition means heat flow and electric current can not penetrate the crack, normal component of the heat flow and electric current at the crack surface are zero. If the distance between the two surfaces of the crack is relative large, and the crack is filled with poor thermal conductivity and electric conductivity media, this boundary condition will have a good approximation.

$$
\begin{gathered}
J_{y}=0, \text { on } S \\
J_{Q y}=0 \text { on } S .
\end{gathered}
$$


Case 2, electrically permeable while thermally impermeable crack. Like the electrically permeable crack used in piezoelectric materials (Jackson [26]), this boundary condition means solid thermal conduction can not penetrate the crack, while the electric current can penetrate the crack, normal component of the solid thermal conduction at the crack surface are zero, while the electric current function $\varphi+\varepsilon T$ and normal component of the electric current at the crack surface are continuous. If the distance between the two surfaces of the crack is small, electrons can penetrate cracks through the tunnel effect, and this boundary condition is more appropriate. In this case, it is necessary that the electric current density $J_{y}$, and electric current function $\varphi+\varepsilon T$ are continuous across the crack surface. On the other hand, the first term of the heat flux $T \varepsilon J$ can not be prevented as the electric current flow through cracks accessible therefore, the thermally impermeable condition means temperature gradient disappears at the crack surface.

$$
\begin{aligned}
J_{y}^{+} & =J_{y}^{-} \text {on } S \\
(\varphi+\varepsilon T)^{+} & =(\varphi+\varepsilon T)^{-} \text {on } S \\
\frac{\partial T}{\partial y} & =0 \text { on } S .
\end{aligned}
$$

\subsection{Infinity conditions}

Considering the electric current condition and the current independent thermal condition at infinity yields

$$
\begin{gathered}
\lim _{z \rightarrow \infty}-\sigma f^{\prime}(z)=J_{x}^{\infty}-i J_{y}^{\infty}, \\
\lim _{z \rightarrow \infty}-2 \kappa f_{2}^{\prime}(z)=J_{Q x}^{\infty}-i J_{Q y}^{\infty} .
\end{gathered}
$$

\subsection{Solution for Case 1 - thermally and electrically imperme-} able crack

According to Eq. (13) and the electric current condition at infinity, analytic function $f(z)$ could be expressed, as:

$$
f(z)=A z+f_{0}(z) .
$$


The following transform function Muskhelishvili [22]:

$$
z=\frac{a}{2}\left(w+\frac{1}{w}\right), \quad w=\omega_{1}[z]=\frac{z+z \sqrt{1-\frac{a^{2}}{z^{2}}}}{a},
$$

maps the crack (with length $2 a$ ) in the z-plane into a unit circle without points $(-1,0)$ and $(1,0)$ in the $w$-plane (Fig. 1.(a), (b)).

Therefore Eq. (13) can be rewrite as:

$$
J_{x}-i J_{y}=-\sigma f^{\prime}(w) \frac{d w}{d z},
$$

and analytic functions $f(w)$ and $f^{\prime}(w)$ could be expressed, as:
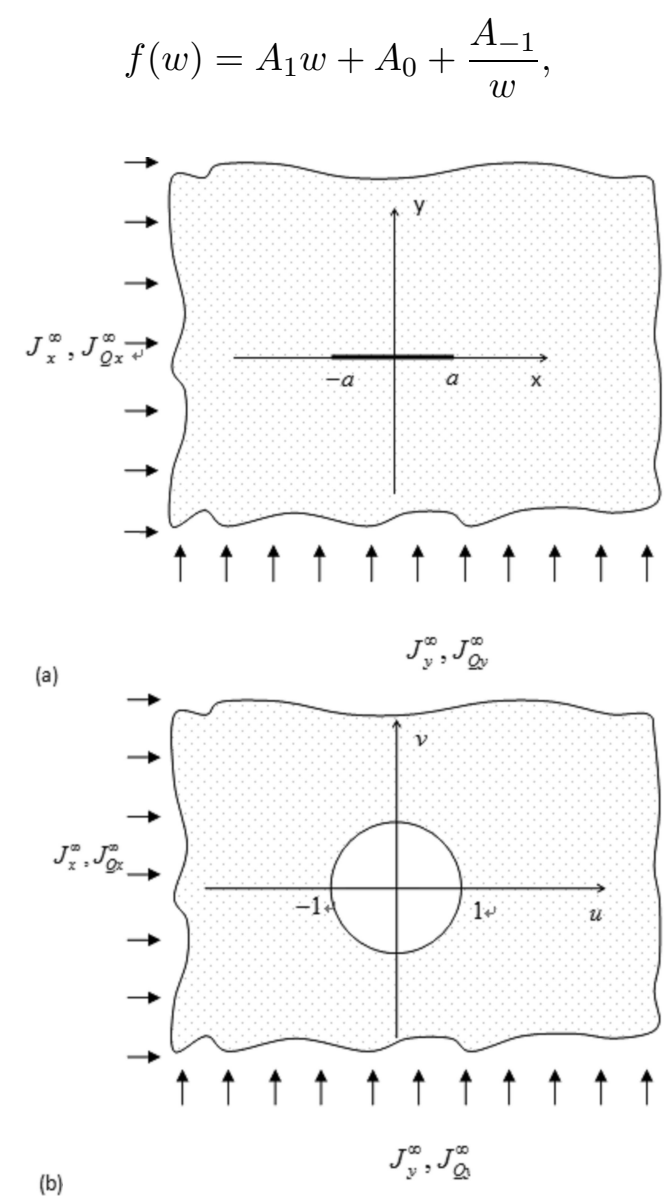

Fig. 1. (a) a crack embedded in a infinite thermoelectric matrix (b) the $w$-plane after conformal mapping 


$$
f^{\prime}(w)=A_{1}-\frac{A_{-1}}{w^{2}} .
$$

Substituting Eq. (32) and Eq. (29) into boundary condition Eq.(21), we have:

$$
f^{\prime}(w) w^{2}+\overline{f^{\prime}(w)}=0 .
$$

Solving Eq. (33) by using Eq. (32), yields:

$$
A_{1}=\bar{A}_{-1} \text {. }
$$

Substituting Eqs (33-34) into boundary condition Eq. (22), we have:

$$
\kappa\left[f_{2}^{\prime}(w) \frac{2 w^{2}}{a}+\overline{f_{2}^{\prime}(w)} \frac{2}{a}\right]=0 .
$$

Letting:

$$
f_{2}(w)=B_{2} w^{2}+B_{1} w+\frac{B_{-1}}{w}+\frac{B_{-2}}{w^{2}} .
$$

Combining it with Eq. (35), and using series expansions, with which a comparison of the coefficients of the same power terms, yields:

$$
B_{2}=\bar{B}_{-2}=0,
$$

$$
B_{-1}=\bar{B}_{1} \text {. }
$$

Considering the infinity condition Eqs (26-27), yields:

$$
\begin{aligned}
A_{1} & =-\frac{a}{2 \sigma}\left(J_{x}^{\infty}-i J_{y}^{\infty}\right), \\
B_{1} & =-\frac{a}{4 \kappa}\left(J_{Q x}^{\infty}-i J_{Q y}^{\infty}\right) .
\end{aligned}
$$

All the coefficients $A_{1}, \bar{A}_{-1}, B_{1}, B_{-1}, B_{2}, B_{-2}$ can be determined so far.

Finally, the temperature field and electric potential field can be expressed as:

$$
\begin{aligned}
& T=-\frac{\sigma}{4 \kappa} f(z) \overline{f(z)}+f_{2}(z)+\overline{f_{2}(z)}+N, \\
& =-\frac{\sigma}{4 \kappa}\left(A_{1} w+\frac{A_{-1}}{w}\right)\left(\bar{A}_{1} \bar{w}+\frac{\bar{A}_{-1}}{\bar{w}}\right)+B_{1} w+\frac{B_{-1}}{w}+\bar{B}_{1} \bar{w}+\frac{\bar{B}_{-1}}{\bar{w}}+N,
\end{aligned}
$$




$$
\varphi=\frac{1}{2}[f(z)+\overline{f(z)}]-\varepsilon T=\frac{1}{2}\left(A_{1} w+\frac{A_{-1}}{w}+\bar{A}_{1} \bar{w}+\frac{\bar{A}_{-1}}{\bar{w}}\right)-\varepsilon T .
$$

4.4. Solution for Case 2- electrically permeable, while thermally impermeable crack

Using the similar method, for electrically permeable while thermally impermeable crack, we have

$$
\begin{gathered}
A_{1}=-\frac{a}{2 \sigma}\left(J_{x}^{\infty}-i J_{y}^{\infty}\right), \\
B_{1}=-\frac{a}{4 \kappa}\left(J_{Q x}^{\infty}-i J_{Q y}^{\infty}\right), \\
A_{-1}=-\frac{a}{2 \sigma}\left(J_{x}^{\infty}-i J_{y}^{\infty}\right), \\
B_{-1}=-\frac{a}{4 \kappa}\left(J_{Q x}^{\infty}+i J_{Q y}^{\infty}\right), \\
B=\frac{a i J_{y}^{\infty}}{\sigma} .
\end{gathered}
$$

\section{Field Intensity Factors}

The behaviour of materials near the crack tip has significant effect on the fracture process. Griffith [27] found that the stress field at a sharp crack tip has a $r^{-1 / 2}$ singularity, which leads to the establishment of the stress intensity factor. The elastic-electric field at crack tip in piezoelectric material also exhibit $r^{-1 / 2}$ singularity (Suo et. al. [28] and Zhang et. al. [29]), which greatly simplifies the elastic-electric field analysis in cracked piezoelectric material. This motivates us to discuss the thermal-electric field at the crack tip in thermoelectric materials.

For the thermally and electrically impermeable crack, the electric current density at the right tip of the crack is:

$$
J_{y}=-\frac{\sigma i}{2}\left[f^{\prime}(z)-\overline{f^{\prime}(z)}\right]=J_{y}^{\infty}+\frac{a J_{y}^{\infty}}{\sqrt{2 a(z-a)}} .
$$

It can be seen, that the electric current density at the crack tip has a $r^{-1 / 2}$ singularity. 
The heat flux at the right tip of the crack is:

$$
\begin{aligned}
J_{Q x}-i J_{Q y}=\frac{\sigma^{2} \varepsilon}{k} f(a) \overline{f(a)} & f^{\prime}(a)-\sigma \varepsilon C_{2} f^{\prime}(a) \\
& +2 \sigma f^{\prime}(a) \overline{f(a)}-2 \sigma \varepsilon f^{\prime}(a) \operatorname{Re}\left[f_{2}(a)\right]-2 \kappa f_{2}^{\prime}(a),
\end{aligned}
$$

where: $f(a)=-\frac{1}{\sigma}\left(J_{x}^{\infty}-i J_{y}^{\infty}\right) a-\frac{a i J_{y}^{\infty}}{\sigma}, f^{\prime}(a)=-\frac{1}{\sigma}\left(J_{x}^{\infty}-i J_{y}^{\infty}\right)+\frac{a i J_{y}^{\infty}}{\sigma \sqrt{2 a(z-a)}}$, $f_{2}(a)=-\frac{a J_{Q x}^{\infty}}{2 \kappa}, f_{2}^{\prime}(a)=\frac{a i J_{Q y}^{\infty}}{2 \kappa} \frac{1}{\sqrt{2 a(z-a)}}$.

It can be seen, that the heat flux at the crack tip also has a $r^{-1 / 2}$ singularity.

For the thermally impermeable and electrically permeable crack, the electric current density at the right tip of the crack is:

$$
J_{y}=-\frac{\sigma i}{2}\left[f^{\prime}(z)-\overline{f^{\prime}(z)}\right]=J_{y}^{\infty} .
$$

It can be seen that the electric current density at the crack tip has no singularity.

The heat flux at the right tip of the crack is:

$$
\begin{aligned}
J_{Q x}-i J_{Q y}=\frac{\sigma^{2} \varepsilon}{k} f(a) \overline{f(a)} f^{\prime}(a)-\sigma \varepsilon C_{2} f^{\prime}(a) \\
+2 \sigma f^{\prime}(a) \overline{f(a)}-2 \sigma \varepsilon f^{\prime}(a) \operatorname{Re}\left[f_{2}(a)\right]-2 \kappa f_{2}^{\prime}(a),
\end{aligned}
$$

where: $f(a)=-\frac{a}{\sigma}\left(J_{x}^{\infty}-i J_{y}^{\infty}\right), f^{\prime}(a)=-\frac{1}{\sigma}\left(J_{x}^{\infty}-i J_{y}^{\infty}\right), f_{2}(a)=-\frac{a J_{Q x}^{\infty}}{2 \kappa}$, $f_{2}^{\prime}(a)=\frac{a i J_{Q y}^{\infty}}{2 \kappa} \frac{1}{\sqrt{2 a(z-a)}}$.

It can be seen, that the heat flux at the crack tip has a $r^{-1 / 2}$ singularity.

\section{Results and discussion}

Numerical calculations were carried out for $\mathrm{Ag}\left(\mathrm{Pb}_{1-y} \mathrm{Sn}_{y}\right)_{m} \mathrm{SbTe}_{2+m}$. Therefore, the material constants of the matrix are: $\varepsilon_{1}=200 \times 10^{-6} \mathrm{~V} / \mathrm{K}$, $\sigma_{1}=110 \times 10^{3} \mathrm{~S} / \mathrm{m}, \kappa_{1}=1.6 \mathrm{~W} / \mathrm{m} / \mathrm{K}$. The calculation area is: $-0.01 \leq$ $x \leq 0.01,-0.01 \leq y \leq 0.01$. The thermoelectric conversion efficiency is $\eta=$ $\frac{J_{Q, \text { input }}-J_{Q, \text { output }}}{J_{Q, \text { input }}}$. 


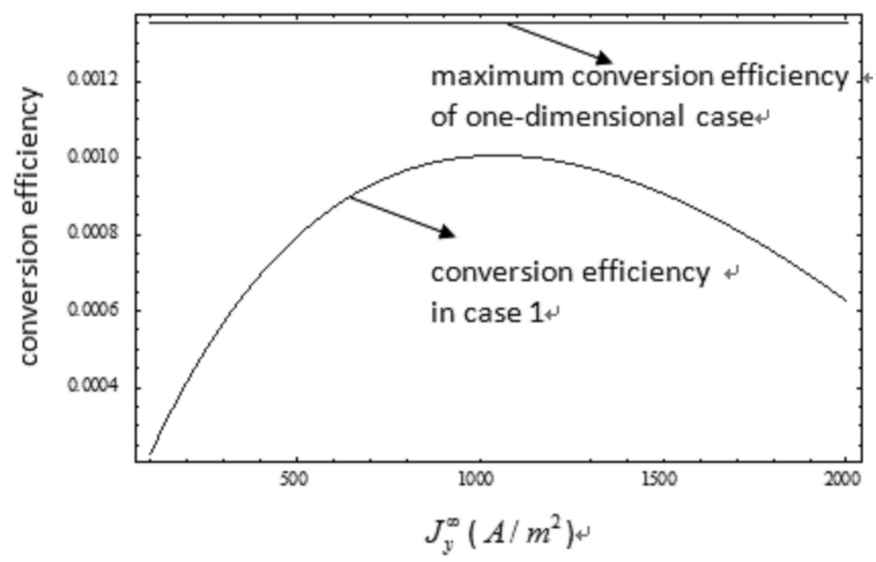

Fig. 2. The conversion efficiency $\eta$ versus electric current density $J_{y}^{\infty}$ in case 1

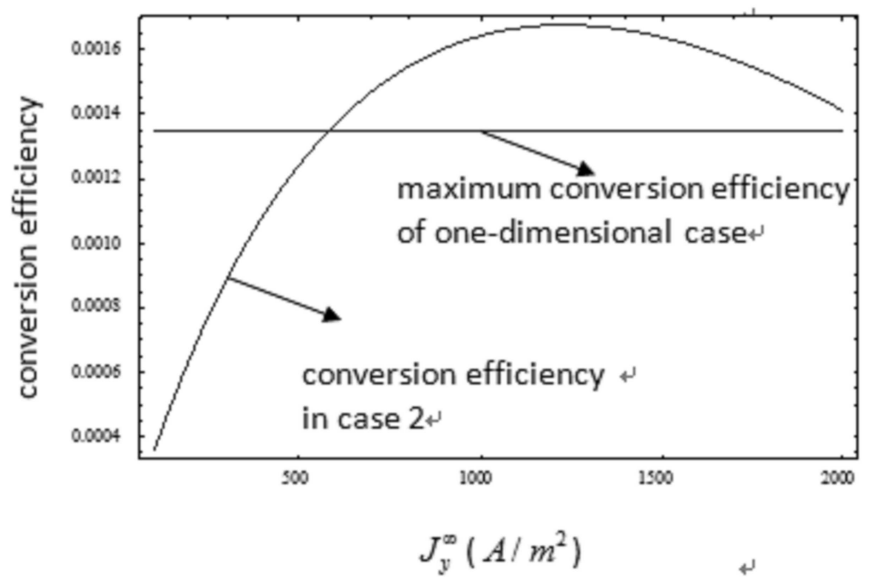

Fig. 3. The conversion efficiency $\eta$ versus electric current density $J_{y}^{\infty}$ in case 2

Figure 2 shows the thermoelectric conversion efficiency $\eta$ versus electric current density $J_{y}^{\infty}$ in case 1 (thermally and electrically impermeable crack) at $a=0.009 \mathrm{~m}, J_{x}^{\infty}=0, J_{Q x}^{\infty}=0$ and. It is seen, that the conversion efficiency $\eta$ firstly increased then decreased with the electric current density $J_{y}^{\infty}$ increasing. Which means, there is an optimal value of electric current density. The thermoelectric conversion efficiency $\eta$ in this case is always less than the maximum conversion efficiency of one-dimensional thermoelectric, under the same temperature condition. 


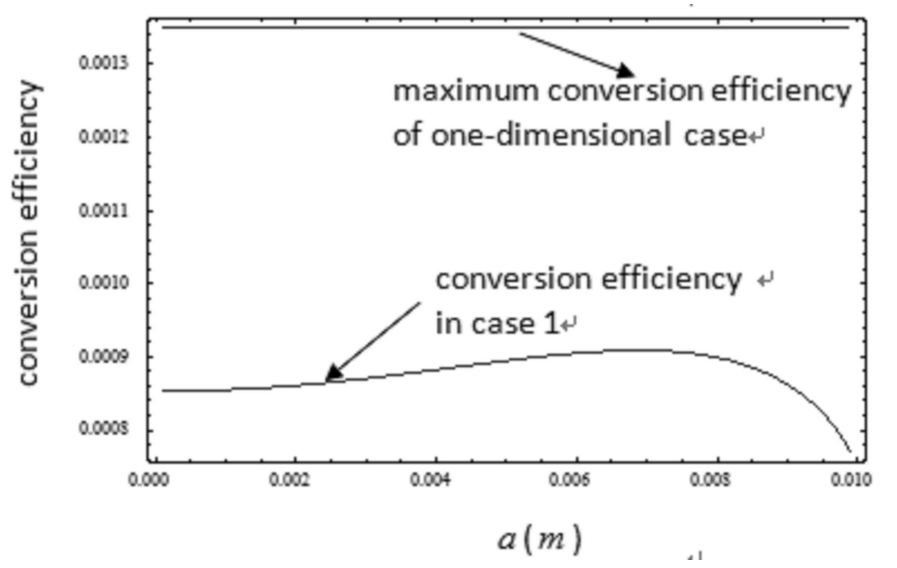

Fig. 4. The conversion efficiency $\eta$ versus crack length $a$ in case 1

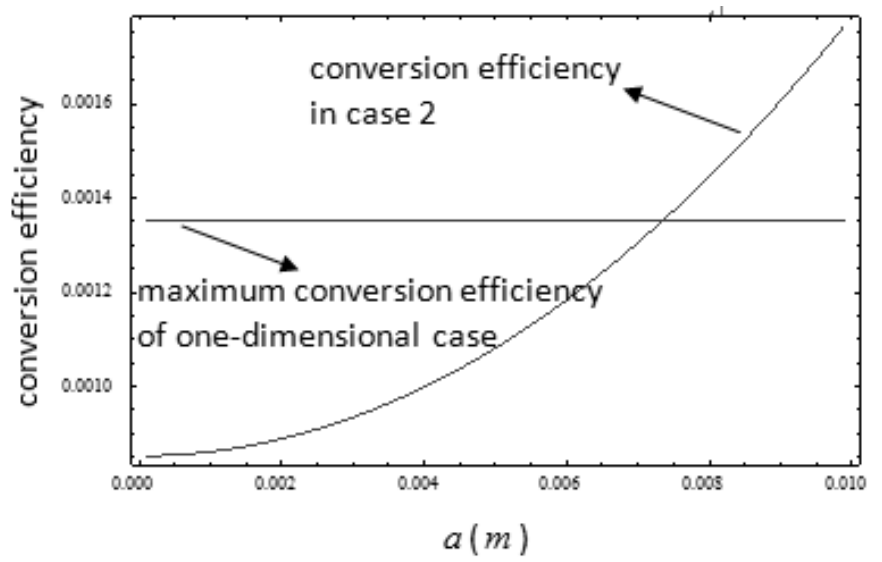

Fig. 5. The conversion efficiency $\eta$ versus crack length $a$ in case 2

The curves for variations of thermoelectric conversion efficiency $\eta$, with respect to electric current density $J_{y}^{\infty}$, in case 2 (thermally impermeable and electrically permeable crack) are shown in Fig. 3 for the case of $a=0.009 \mathrm{~m}$, $J_{x}^{\infty}=0, J_{Q x}^{\infty}=0$ and $J_{Q y}^{\infty}=200 \mathrm{~J} / \mathrm{m}^{2} \cdot \mathrm{s}$. It is seen, that the thermoelectric conversion efficiency $\eta$ in this case can be higher than the maximum conversion efficiency of one-dimensional thermoelectric under the same temperature condition. This is important, as it proves the maximum conversion efficiency of one-dimensional thermoelectric may be a breakthrough in the two-dimensional condition.

The conversion efficiency improvement is due to the electric current 
flow and thermal flux separation. Heat flow presence around the thermally impermeable and electrically permeable crack, is resulting in increased heat flow path. Under the same temperature difference, an increased heat flow path reduces the temperature gradient along the path, therefore reducing the heat leak and improving the conversion efficiency.

Figures 4-5 show the thermoelectric conversion efficiency $\eta$ versus crack length $a$ in case 1 , (Figure 4) and case 2, (Figure 5) at $J_{y}^{\infty}=1600 \mathrm{~A} / \mathrm{m}^{2}, J_{x}^{\infty}=$ $0, J_{Q x}^{\infty}=0$ and $J_{Q y}^{\infty}=200 \mathrm{~J} / \mathrm{m}^{2} \cdot \mathrm{s}$. It is seen, that the thermoelectric conversion efficiency $\eta$ in case 1 is always less than the maximum conversion efficiency of one-dimensional thermoelectric under the same temperature condition. While the thermoelectric conversion efficiency $\eta$ in case 2 can be higher than the maximum conversion efficiency of one-dimensional thermoelectric under the same temperature condition. The conversion efficiency in case 2 increases with increasing crack length $a$, the maximum conversion efficiency is 0.00175 , which is increased by $29.6 \%$ compared to the maximum conversion efficiency of onedimensional thermoelectric under the same temperature condition.

\section{Conclusions and future work}

The crack problem in a thermoelectric material is studied in this paper. The closed form solutions are derived. The solution show, that the electric current density and thermal flux density at the crack tip have a $r^{-1 / 2}$ singularity in case 1 , while the electric current density at the crack tip has no singularity in case 2. Due to the electric current flow and thermal flux separation, the thermoelectric conversion efficiency $\eta$ in case 2 can be higher than the onedimensional thermoelectric under the same temperature condition.

In the future, we will continue to study the coupled thermo-elastoelectric problem and inclusion problem in thermoelectric material, and we are here to give some discussions.

For thermal stress problems, stress function $\Phi$ must satisfy the compatibility equation:

$$
\nabla^{4} \Phi+E \alpha \nabla^{2} T=0
$$

where $\alpha$ is the thermal expansion coefficient. This results in:

$$
\nabla^{4} \Phi=-E \alpha \nabla^{2} T=\frac{E \alpha}{\sigma \kappa} \mathbf{J} \bullet \mathbf{J}=\frac{E \alpha \sigma}{\kappa} f^{\prime}(z) \overline{f^{\prime}(z)} .
$$

Therefore, stress functions $\Phi$ are composed by general solution and particular solution:

$$
\Phi=\Phi_{1}+\Phi_{2}
$$


where the general solution $\Phi_{2}$ satisfies $\nabla^{4} \Phi_{2}=0$, and the particular solution $\Phi_{1}$ satisfies $\nabla^{4} \Phi_{1}=\frac{E \alpha \sigma}{\kappa} f^{\prime}(z) \overline{f^{\prime}(z)}$. The particular solution could be chosen as: $\Phi_{1}=-\frac{E \alpha}{4} \iint T d z d \bar{z}$, as the temperature can be obtained by the method developed in this paper, the subsequent task is to search the general solution $\Phi_{2}$ (satisfies $\nabla^{4} \Phi_{2}=0$ ) at given stress boundary conditions, which is similar to the pure elastic problem.

For inclusion problems, there will be four unknown functions, $f(z)$, $f_{2}(z)$ in matrix and $f_{*}(z), f_{2 *}(z)$ in inclusion. Correspondingly, we will have four boundary conditions, such as the temperature, electric potential, electric current density, and heat flux, are continuous across the interface. The inclusion problem may be solved substituting the four unknown functions into the four boundary conditions and using the similar mathematical treatment as used in Sec. 4.

\section{REFERENCES}

[1] Disalvo, F. J. Thermoelectric Cooling and Power Generation. Science, 285 (1999), 703-706.

[2] Yang, J. H., T. Caillat. Thermoelectric Materials for Space and Automotive Power Generation. MRS Bulletin, 31 (2006), 224229.

[3] Narducci, D. Do We Really Need High Thermoelectric Figures of Merit? A Critical Appraisal to the Power Conversion Efficiency of Thermoelectric Materials. Appl. Phys. Lett., 99 (2011), 102-104.

[4] Tritt, T. M., M. A. Subramanian. Thermoelectric Materials, Phenomena, and Applications: A Bird's Eye View. MRS Bulletin, 31 (2006), 188-194.

[5] Kraemer, D., B. Poudel, H. P. Feng, J. C. Caylor, B. Yu, X. Yan, Y. Ma, X. W. Wang, D. Z. Wang, A. Muto, K. McEnaney, M. Chiesa, Z. F. Ren, G. Chen. High Performance Flat-panel Solar Thermoelectric Generators with High Thermal Concentration. Nature Materials, 10 (2011), 532-538.

[6] Bell, L. E. Cooling, Heating, Generating Power, and Recovering Waste Heat with Thermoelectric Systems. Science, 321 (2008), 1457-1461.

[7] Heremans, J. P., C. M. Thrush, D. T. Morelli, M. C. Wu. Thermoelectric Power of Bismuth Nanocomposites. Physical Review Letters, 88 (2002), 216801.

[8] Snyder, G. J., E. S. Toberer. Complex Thermoelectric Materials. Nature Materials, 7 (2008), 105-114.

[9] Gothard, N., X. Ji, J. He, T. M. Tritta. Thermoelectric and Transport Properties of N-type Bi2Te3 Nanocomposites. Journal of Applied Physics, 103 (2008), 054314. 
[10] Vashaee, D., A. Shakouri. Improved Thermoelectric Power Factor in Metalbased Superlattices. Physical Review Letters, 92 (2004), 106103.

[11] Webman, I., J. Jortner, M. H. Cohen. Thermoelectric-Power in Inhomogeneous Materials. Physical Review B, 16 (1977), 2959-2964.

[12] Mahan, G. D. Parallel Thermoelectrics. Physical Review B, 87 (2013), 045415.

[13] Isoda, Y., Y. Shinohara, Y. Imai, I. Nishida, O. Ohashi. Thermal Shock Resistance and Thermoelectric Properties of Boron Doped Tron Disilicides. J. Jpn. Inst. Met., 63 (1999), 391-396.

[14] Zhao, D. G., C. W. Tian, Y. T. Liu, C. W. Zhan, L. D. Chen. High Temperature Sublimation Behaviour of Antimony in CoSb3 Thermoelectric Material during Thermal Duration Test. J. Alloy. Compd., 509 (2011), 3166-3171.

[15] Schmidt, R. D., E. D. Case, J. Gilesili, J. E. Ni, T. P. Hogan. Roomtemperature Mechanical Properties and Slow Crack Growth Behaviour of $\mathrm{Mg}_{2} \mathrm{Si}$ Thermoelectric Materials. J. Electron. Mater, 41 (2012), 1210-1216.

[16] Eilertsen, J., M. A. Subramanian, J. J. Kruzic. Fracture Toughness of $\mathrm{Co}_{4} \mathrm{Sb}_{12}$ and $\mathrm{In}_{0.1} \mathrm{Co}_{4} \mathrm{Sb}_{12}$ Thermoelectric Skutterudites evaluated by Three Methods. J. Alloy. Compd., 552 (2013), 492-498.

[17] Hu, K. F., S. Loo, F. Guo, W. Chen, J. S. Dyck, C. Uher, T. Hogan, E. K. Polychroniadis, M. G. Kanatzidis. Cubic AgPbmSbTe ${ }_{2+m}$ : Bulk Thermoelectric Materials with High Figure of Merit. Science, 303 (2004), 818821.

[18] Poudel, B., Q. Hao, Y. Ma, Y. C. Lan, A. Minnich, B. Yu, X. Yan, D. Z. Wang, A. Muto, D. Vashaee, X. Y. Chen, J. M. Liu, M. S. DresselhaU, G. Chen, Z. F. Ren. High Thermoelectric Performance of Nanostructured Bismuth Antimony Telluride Bulk Alloys. Science, 320 (2008), 634-638.

[19] Cao, Y. Q., X. B. Zhao, T. J. Zhu, X. B. Zhang, J. P. Tu. Syntheses and Thermoelectric Properties of $\mathrm{Bi}_{2} \mathrm{Te}_{3} / \mathrm{Sb}_{2} \mathrm{Te}_{3}$ Bulk Nanocomposites with Laminated Nanostructure. Appl. Phys. Lett., 92 (2008), 143106.

[20] Harman, T. C., J. M. Honig. Thermoelectric and Thermomagnetic Effects and Applications, New York, McGraw-Hill, 1967.

[21] Yang, Y., F. Y. Ma, C. H. Lei, Y. Y. LiU, J. Y. Li. Nonlinear Asymptotic Homogenization and the Effective Behaviour of Layered Thermoelectric Composites. Journal of the Mechanics and Physics of Solids, 61 (2013), 1768-1783.

[22] Muskhelishvili, N. L. Some Basic Problems of Mathematical Theory of Elasticity, Noordhoff, Leyden, 1975.

[23] Irwin, G. Analysis of Stresses and Strains Near the End of a Crack Traversing a Plate. Journal of Applied Mechanics, 24 (1957), 361-364.

[24] Deeg, W. F. The Analysis of Dislocation, Crack and Inclusion Problems in Piezoelectric Solids, Ph. D. Thesis, Stanford University, 1980.

[25] PAK, Y. E. Linear Electroelastic Fracture Mechanics of Piezoelectric Materials. Int. J. Fract., 54 (2002), 79-100.

[26] Jackson, J. D. Classical Electrodynamics. New York, John Wiley, 1976. 
[27] Griffith, A. A. The Phenomena of Rupture and Flow in Solids. Philosophical Transactions of the Royal Society of London, A, 221 (1921), 163-198.

[28] Suo, Z., C. M. Kuo, D. M. Barnett, J. R. Willis. Fracture Mechanics for Piezoelectric Ceramics. J. Mech. Phys. Solids, 40 (1992), 739-765.

[29] Zhang, T. Y., T. H. Wang, M. H. Zhao. Interaction of a Piezoelectric Screw Dislocation with an Insulating Crack. Philos. Mag. A, 82 (2002), 2805-2824.

\section{Nomenclature}

$a$ semi-length of the crack, $\mathrm{m}$

$\mathbf{J}$ electric current density, $\mathrm{A} / \mathrm{m}^{2}$

$\mathbf{J}_{E}$ energy flux, $\mathrm{J} / \mathrm{m}^{2} \cdot \mathrm{s}$

$\mathbf{J}_{Q}$ heat flux, $\mathrm{J} / \mathrm{m}^{2} \cdot \mathrm{s}$

$T$ temperature, $\mathrm{K}$

$e$ charge, $\mathrm{C}$

$u$ axial coordinate in the $w$-plane, $\mathrm{m}$

$v$ axial coordinate in the $w$-plane, $\mathrm{m}$

$x$ axial coordinate, $\mathrm{m}$

$y$ axial coordinate, $\mathrm{m}$

$z$ complex coordinate, $\mathrm{m}$

$\sigma$ electric conductivity, $\mathrm{S} / \mathrm{m}$

$\kappa$ thermal conductivity, $\mathrm{W} / \mathrm{m} \cdot \mathrm{K}$

$\phi$ electric potential, $V$

$A_{k}$ unknown constant

$B_{k}$ unknown constant

$C_{2}$ unknown constant

Re real part

Im imaginary part

$i$ unit imaginary number

$\nabla$ gradient

$\nabla^{2}$ divergence of the gradient 